\title{
ANALYSIS OF HOM INTERACTION BETWEEN CAVITIES BY MULTIMODAL S-PARAMETER MEASUREMENTS
}

\author{
H.-W. Glock, F. Marhauser, P. Hülsmann, M. Kurz, H. Klein \\ Institut für Angewandte Physik der Johann Wolfgang Goethe-Universität Frankfurt am Main \\ Robert Mayer-Straße 2-4, D-60054 Frankfurt/M., Germany
}

\begin{abstract}
Accelerating cavities exchange HOM power through interconnecting beam pipes in case of signal frequencies above the cut-off of their propagating waveguide modes. This may lead either to improved HOM damping or - in the case most severe - to unwanted phase coherence of fields to the beam. Therefore the knowledge of the scattering properties of a cavity as a line element is needed to analyse all kinds of RF cavity-cavity interaction. Since there is a lack of measurement tools capable to provide a multidimensional scattering matrix at a given frequency point, we have been developing a method for this purpose. It uses a set of 2-port S-parameters of the device under test, embedded in a number of geometrically different RF environments. The application of the method is demonstrated with copper models of TESLA cavities.
\end{abstract}

\section{INTRODUCTION}

Usually the spectra of beam-excited fields contain significant parts above the RF cut-off of several waveguide modes of the beam pipe. Therefore one has to be concerned about the effects caused by the RF-interaction of the beam line elements: it may for example increase the damping of resonant cavity modes by stronger coupling to absorbing elements, it allows for resonant fields localised between different beam pipe insertions or it may introduce signal paths delayed by multiple reflection to - in the worst case - particle velocity. Either of these situations is covered within a description in terms of transmission and reflection properties of the beam line's elements with respect to their interconnecting planes, i.e. their scattering (S-) parameters. Since the interaction is not restricted to the fundamental mode, but includes a - frequency dependent - number of waveguide modes, the multidimensional matrix of the S-parameters (see sect. 2) is necessary and sufficient to characterise a line element in any environment. Thus we have been developing an experimental method, allowing for multidimensional S-matrix measurement in a waveguide setup. Since its first presentations $[1,2]$ we adopted an improved evaluation scheme which minimizes the overall error. It is demonstrated in the case of two modes for a device measurement and three modes for a calibration of an adaptor at single frequency points.

\section{THEORY}

The S-matrix of an adaptor (A, B in Fig. 1) with one coaxial line (index 0 ) and $n$ modes propagating in the waveguide port may be written as:

$$
\underline{\underline{\mathrm{A}}}=\left(\begin{array}{c|ccc}
\mathrm{A}_{00} & \mathrm{~A}_{01} & \cdots & \mathrm{A}_{0 \mathrm{n}} \\
\hline \mathrm{A}_{01} & \mathrm{~A}_{11} & \cdots & \mathrm{A}_{1 \mathrm{n}} \\
\vdots & \vdots & \ddots & \vdots \\
\mathrm{A}_{0 \mathrm{n}} & \mathrm{A}_{1} \mathrm{n} & \cdots & \mathrm{A}_{\mathrm{n} n}
\end{array}\right)=\left(\begin{array}{c|c}
\mathrm{A}_{00} & \overrightarrow{\mathrm{A}}^{\mathrm{T}} \\
\hline \overrightarrow{\mathrm{A}} & \underline{\underline{\mathrm{A}}}
\end{array}\right)
$$

Herein the scalar $\mathrm{A}_{00}$ describes the reflection at the coaxial port, $\vec{A}$ the coupling from the coaxial line to each waveguide mode and $\mathrm{A}$ the reflection at the waveguide flange, that may couple every mode to each other. The matrix is symmetric due to the reciprocity of the device. Like in the single mode case, the problem of determining the properties of a device splits into the calibration step - i.e. determination of the adaptors, which connect the device to an ideal network analyzer - and the measurement once the adaptors are known. Considering the number of unknowns (10 for two modes at two waveguide ports) it becomes clear that a single measurement with two completely known adaptors, which gives three numbers (two reflection, one transmission quantity), not provides sufficient information. Thus one has to use different pairs of known adaptors for a number of subsequent device measurements. To keep the calibration effort as small as possible we take two adaptors and then combine them with various delay line lengths (Fig. 1). In the same manner we use a short and different delay line lengths (which are the only reliable broadband waveguide standards of easy making) to calibrate the two adaptors.
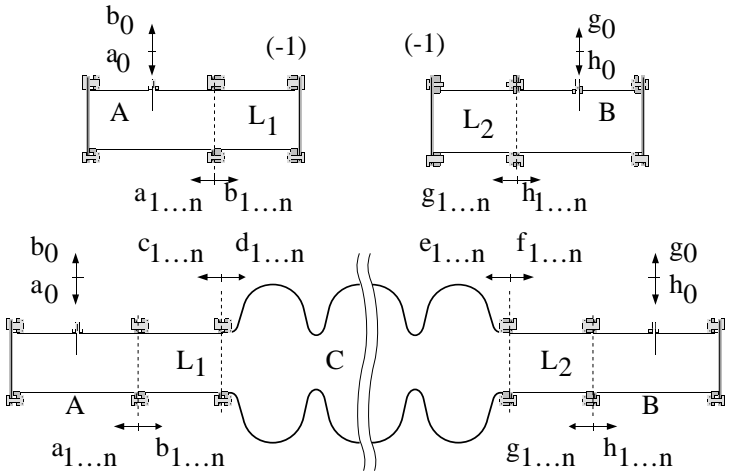

Figure 1: Schematic drawing of setups used for adaptor calibration with delayed shorts and for measurement. Small letters denote the signals at all connection planes, index 0 corresponds to the coaxial line.

If we consider a setup with two adaptors $\mathrm{A}$ and $\mathrm{B}$, a test device $\mathrm{C}$ and two connecting waveguides with lengths $\mathrm{L}_{1}$ and $L_{2}$ (Fig. 1), we are able to write down all signals, related by S-matrices: 


$$
\begin{aligned}
& \left(\begin{array}{c}
\mathrm{b}_{0} \\
\overrightarrow{\mathrm{b}}
\end{array}\right)=\left(\begin{array}{cc}
\mathrm{A}_{00} & \overrightarrow{\mathrm{A}}^{\mathrm{T}} \\
\overrightarrow{\mathrm{A}} & \underline{\underline{\mathrm{A}}}
\end{array}\right)\left(\begin{array}{c}
\mathrm{a}_{0} \\
\overrightarrow{\mathrm{a}}
\end{array}\right),\left(\begin{array}{c}
\mathrm{g}_{0} \\
\overrightarrow{\mathrm{g}}
\end{array}\right)=\left(\begin{array}{cc}
\mathrm{B}_{00} & \overrightarrow{\mathrm{B}}^{\mathrm{T}} \\
\overrightarrow{\mathrm{B}} & \underline{\underline{\mathrm{B}}}
\end{array}\right)\left(\begin{array}{c}
\mathrm{h}_{0} \\
\overrightarrow{\mathrm{h}}
\end{array}\right) \\
& \left(\begin{array}{l}
\overrightarrow{\mathrm{a}} \\
\overrightarrow{\mathrm{d}}
\end{array}\right)=\left(\begin{array}{cc}
0 & \underline{\underline{E}}\left(\mathrm{~L}_{1}\right) \\
\underline{\underline{E}\left(\mathrm{~L}_{1}\right)} & 0
\end{array}\right)\left(\begin{array}{l}
\overrightarrow{\mathrm{b}} \\
\overrightarrow{\mathrm{c}}
\end{array}\right),\left(\begin{array}{l}
\overrightarrow{\mathrm{e}} \\
\overrightarrow{\mathrm{h}}
\end{array}\right)=\left(\begin{array}{cc}
0 & \underline{\underline{E}}\left(\mathrm{~L}_{2}\right) \\
\underline{\underline{E}}\left(\mathrm{~L}_{2}\right) & 0
\end{array}\right)\left(\begin{array}{l}
\overrightarrow{\mathrm{f}} \\
\overrightarrow{\mathrm{g}}
\end{array}\right) \\
& \left(\begin{array}{l}
\overrightarrow{\mathrm{c}} \\
\overrightarrow{\mathrm{f}}
\end{array}\right)=\left(\begin{array}{ll}
\underline{\mathrm{C}}_{11} & \underline{\underline{\mathrm{C}}}_{12} \\
\underline{\underline{\mathrm{C}}}_{12}^{\mathrm{T}} & \underline{\underline{\mathrm{C}}}_{22}
\end{array}\right)\left(\begin{array}{l}
\overrightarrow{\mathrm{d}} \\
\overrightarrow{\mathrm{e}}
\end{array}\right)
\end{aligned}
$$

All the submatrices are $(\mathrm{n} \times \mathrm{n})$-dimensional. For the waveguide of length $L$ and the phase constants $\gamma_{j}$ holds:

$\underline{\underline{E}}(\mathrm{~L})=\left(\begin{array}{ccc}\mathrm{e}^{-\gamma_{1}} \mathrm{~L} & 0 & 0 \\ 0 & \ddots & 0 \\ 0 & 0 & \mathrm{e}^{-\gamma_{\mathrm{n}}} \mathrm{L}\end{array}\right)$

Using an additional abbreviation

$$
\left(\begin{array}{l}
\underline{\underline{\mathrm{H}}}_{11} \underline{\underline{\mathrm{H}}}_{12} \\
\underline{\underline{\underline{\mathrm{H}}}}_{12}^{\mathrm{T}} \underline{\underline{\underline{H}}}_{22}
\end{array}\right)=\left(\begin{array}{cc}
\underline{\underline{\mathrm{E}}}\left(\mathrm{L}_{1}\right) & 0 \\
0 & \underline{\underline{\mathrm{E}}}\left(\mathrm{L}_{2}\right)
\end{array}\right)\left(\begin{array}{l}
\underline{\underline{\mathrm{C}}}_{11} \underline{\underline{\mathrm{C}}}_{12} \\
\underline{\underline{\mathrm{C}}}_{12}^{\mathrm{T}} \underline{\underline{\mathrm{C}}}_{22}
\end{array}\right)\left(\begin{array}{cc}
\underline{\underline{\mathrm{E}}}\left(\mathrm{L}_{1}\right) & 0 \\
0 & \underline{\underline{\mathrm{E}}}\left(\mathrm{L}_{2}\right)
\end{array}\right)
$$

and with respect to the fact, that the complete setup is just a coaxial line - two port with a $(2 \times 2)$-S-matrix

$\left(\begin{array}{l}\mathrm{b}_{0} \\ \mathrm{~g}_{0}\end{array}\right)=\left(\begin{array}{ll}\Gamma_{1} & \mathrm{~T} \\ \mathrm{~T} & \Gamma_{2}\end{array}\right)\left(\begin{array}{l}\mathrm{a}_{0} \\ \mathrm{~h}_{0}\end{array}\right)$

one finds after elimination of all signal quantities ([1]):

$$
\begin{aligned}
& \left(\begin{array}{ll}
\Gamma_{1} & \mathrm{~T} \\
\mathrm{~T} & \Gamma_{2}
\end{array}\right)=\left(\begin{array}{cc}
\mathrm{A}_{00} & 0 \\
0 & \mathrm{~B}_{00}
\end{array}\right)+ \\
& +\left(\begin{array}{cc}
\overrightarrow{\mathrm{A}}^{\mathrm{T}} & 0 \\
0 & \overrightarrow{\mathrm{B}}^{\mathrm{T}}
\end{array}\right)\left(\begin{array}{ll}
\underline{\underline{\mathrm{H}}}_{11} & \underline{\mathrm{H}}_{12} \\
\underline{\underline{\underline{H}}}_{12}^{\mathrm{T}} & \underline{\underline{H}}_{22}
\end{array}\right)\left[1-\left(\begin{array}{cc}
\underline{\underline{\mathrm{A}}} & 0 \\
\overline{0} & \underline{\underline{\mathrm{B}}}
\end{array}\right)\left(\begin{array}{ll}
\underline{\underline{H}}_{11} & \underline{\mathrm{H}}_{12} \\
\underline{\underline{\mathrm{H}}}_{12}^{\mathrm{T}} & \underline{\underline{H}}_{22}
\end{array}\right)\right]^{(-1)}\left(\begin{array}{cc}
\overrightarrow{\mathrm{A}} & 0 \\
0 & \overrightarrow{\mathrm{B}}
\end{array}\right)
\end{aligned}
$$

We shall refer to (5) as the "complete model". It can be rewritten using

$$
(\underline{\underline{1}}-\underline{\underline{M}})^{(-1)}=\left(\underline{\underline{1}}+\underline{\underline{\mathrm{M}}}+\underline{\underline{M}}^{2}+\underline{\underline{M}}^{3}+\ldots\right)
$$

(we skip the discussion of the mathematical conditions)

$$
\begin{aligned}
& \left(\begin{array}{ll}
\Gamma_{1} & \mathrm{~T} \\
\mathrm{~T} & \Gamma_{2}
\end{array}\right)=\left(\begin{array}{cc}
\mathrm{A}_{00} & 0 \\
0 & \mathrm{~B}_{00}
\end{array}\right)+ \\
& +\left(\begin{array}{cc}
\overrightarrow{\mathrm{A}}^{\mathrm{T}} & 0 \\
0 & \overrightarrow{\mathrm{B}}^{\mathrm{T}}
\end{array}\right)\left(\begin{array}{ll}
\underline{\underline{\mathrm{H}}}_{11} & \underline{\underline{H}}_{12} \\
\underline{\underline{\mathrm{H}}}_{12}^{\mathrm{T}} & \underline{\underline{H}}_{22}
\end{array}\right) \sum_{\mathrm{j}=0}^{\infty}\left[\left(\begin{array}{ll}
\underline{\underline{\mathrm{A}}} & 0 \\
\overline{0} & \underline{\underline{\mathrm{B}}}
\end{array}\right)\left(\begin{array}{ll}
\underline{\underline{H}}_{11} & \underline{\underline{\mathrm{H}}}_{12} \\
\underline{\underline{\mathrm{H}}}_{12}^{\mathrm{T}} & \underline{\underline{H}}_{22}
\end{array}\right)\right]^{\mathrm{j}}\left(\begin{array}{cc}
\overrightarrow{\mathrm{A}} & 0 \\
0 & \overrightarrow{\mathrm{B}}
\end{array}\right)
\end{aligned}
$$

as a geometric matrix series. This expansion is useful as well as an approach for the numerical solution of (5) with a set of measurement data as for its physical interpretation. We are going to use an approximation for the right hand side of (7), that contains only the terms of order $j=0$ and $\mathrm{j}=1$. This will be called "reduced model". To simplify discussion, we restrict ourselves to the calibration step, which is a special case of (5) (set all elements of $C$ to 0 except the upper left block which is the negative identity). Then the complete model is:

$$
\Gamma_{1}\left(\mathrm{~L}_{1}\right)=\mathrm{A}_{00}-\overrightarrow{\mathrm{A}}^{\mathrm{T}} \underline{\underline{E}}^{2}\left(\mathrm{~L}_{1}\right)\left[\underline{\underline{1}}+\underline{\underline{\mathrm{A}}} \underline{\underline{E}}^{2}\left(\mathrm{~L}_{1}\right)\right]^{(-1)} \overrightarrow{\mathrm{A}}
$$

and its reduced version reads like:

$$
\Gamma_{1}\left(\mathrm{~L}_{1}\right) \approx \mathrm{A}_{00}-\overrightarrow{\mathrm{A}}^{\mathrm{T}} \underline{\underline{E}}^{2}\left(\mathrm{~L}_{1}\right) \overrightarrow{\mathrm{A}}+\overrightarrow{\mathrm{A}}^{\mathrm{T}} \underline{\underline{E}}^{2}\left(\mathrm{~L}_{1}\right) \underline{\underline{\mathrm{A}}} \underline{\underline{E}}^{2}\left(\mathrm{~L}_{1}\right) \overrightarrow{\mathrm{A}}
$$

Evaluating this for two modes (10) shows that each term describes a possible signal path from initial incidence to final detection. Thus the arithmetic derivation of (5) ((7) resp.) is just a summation over all paths, written very compact.

$$
\begin{aligned}
\Gamma_{1}\left(\mathrm{~L}_{1}\right)= & \mathrm{A}_{00}-\left(\mathrm{A}_{01}^{2} \mathrm{e}^{-2 \mathrm{i} \gamma_{1} \mathrm{~L}_{1}}+\mathrm{A}_{02}^{2} \mathrm{e}^{\left.-2 \mathrm{i} \gamma_{2} \mathrm{~L}_{1}\right)+}\right. \\
+ & \left(\mathrm{A}_{01}^{2} \mathrm{~A}_{11} \mathrm{e}^{-4 \mathrm{i} \gamma_{1} \mathrm{~L}_{1}}+\mathrm{A}_{02}^{2} \mathrm{~A}_{22} \mathrm{e}^{-4 \mathrm{i} \gamma_{2} \mathrm{~L}_{1}}+\right. \\
& \left.+2 \mathrm{~A}_{01} \mathrm{~A}_{02} \mathrm{~A}_{12} \mathrm{e}^{-2 \mathrm{i}\left(\gamma_{1}+\gamma_{2}\right) \mathrm{L}_{1}}\right)-\cdots
\end{aligned}
$$

In order to find the S-parameters of C (A, B resp. during calibration), a set of equations (5) ((8) resp.) with measured data inserted has to be solved. This is done by an iteration scheme, which significantly improves the results compared with the approximative approach $[1,2]$. The latter one remains as a first step of the method, used to find initial values in the following way: Neglecting all higher orders of internal reflection but the first, $\Gamma_{1}\left(\mathrm{~L}_{1}, \mathrm{~L}_{2}\right)$ (and similar $\left.\mathrm{T}, \Gamma_{2}\right)$ is a linear combination of length oscillations with well known wavenumbers (left column) and amplitudes with simple dependencies on Sparameters, listed here for the easiest case (10):

\begin{tabular}{|c|c|c|}
\hline 1 & $\mathrm{~A}_{00}$ & $\Rightarrow \mathrm{A}_{00}$ \\
\hline $\mathrm{e}^{-2 \mathrm{i} \gamma_{1} \mathrm{~L}_{1}}$ & $-\mathrm{A}_{01}^{2}$ & $\Rightarrow \pm \mathrm{A}_{01}$ \\
\hline $\mathrm{e}^{-2 \mathrm{i} \gamma_{2} \mathrm{~L}_{1}}$ & $-\mathrm{A}_{02}^{2}$ & $\Rightarrow \pm \mathrm{A}_{02}$ \\
\hline $\mathrm{e}^{-4 \mathrm{i} \gamma_{1} \mathrm{~L}_{1}}$ & $\mathrm{~A}_{01}^{2} \mathrm{~A}_{11}$ & $\Rightarrow \mathrm{A}_{11}$ \\
\hline $\mathrm{e}^{-4 \mathrm{i} \gamma_{2} \mathrm{~L}_{1}}$ & $\mathrm{~A}_{02}^{2} \mathrm{~A}_{22}$ & $\Rightarrow \mathrm{A}_{22}$ \\
\hline $\mathrm{e}^{-2 \mathrm{i}\left(\gamma_{1}+\gamma_{2}\right) \mathrm{L}_{1}}$ & $2 \mathrm{~A}_{01} \mathrm{~A}_{02} \mathrm{~A}_{12}$ & $\Rightarrow \pm \mathrm{A}_{12}$ \\
\hline
\end{tabular}

Thus the amplitudes and consequently the S-parameters are found explicitly from the solution of an overdetermined system of equations like (10) with $\Gamma$ taken from the set of experiments with different L. Although the S-parameters found in this manner seemed to resemble the measured system behaviour quite well [1,2], consistency tests and experiments simulated numerically (sect. 3) showed, that the S-parameters, especially if weakly contributing, may carry intolerable errors. The reason was found to be the influence of higher orders of internal reflection, neglected so far. Therefore their contribution has to be incorporated, which is done by iteration. Again, transform (8) using (6), but keep all higher reflection degrees - again as a geometric series - together with $\Gamma$ on the left hand side:

$$
\Gamma+\left(\overrightarrow{\mathrm{A}}^{\mathrm{T}} \underline{\underline{E}}^{2} \underline{\underline{\mathrm{A}}} \underline{\underline{E}}^{2}\left[\underline{\underline{1}}+\underline{\underline{\mathrm{A}}} \underline{\underline{E}}^{2}\right]^{-1} \underline{\underline{\mathrm{A}}} \underline{\underline{E}}^{2} \overrightarrow{\mathrm{A}}\right)=
$$$$
=\mathrm{A}_{00}-\overrightarrow{\mathrm{A}}^{\mathrm{T}} \underline{\underline{E}}^{2} \overrightarrow{\mathrm{A}}+\overrightarrow{\mathrm{A}}^{\mathrm{T}} \underline{\underline{E}}^{2} \underline{\underline{\mathrm{A}}} \underline{\underline{E^{2}}} \overrightarrow{\mathrm{A}}
$$

The expression in brackets is easily evaluated for given Sparameters and corrects the measured $\Gamma$ for the higher reflection degrees, of course on an approximative base. Then the reduced model on the right is fitted as before to the left hand side, which gives new S-parameters. The procedure is repeated until the variation of the S-parameters vanishes, which corresponds to the best approximation of the complete model to the measured data. All considerations demonstrated for the calibration step (starting from (8)) can be transferred to the measurement (5).

\section{SIMULATED EXPERIMENTS}

In order to test the procedure a 3-mode set of S-parameters of two adaptors $\mathrm{A}, \mathrm{B}$ and a device $\mathrm{C}$ was generated to evaluate (5) for a typical set of $18 \times 18$ different 
combinations of $\mathrm{L}_{1}, \mathrm{~L}_{2}$. This was taken as "measured" input. Tab. 1 shows 4 (of 21) parameters of C; first the true value originally invested, the initial value found by the fit-method and the result of the $4^{\text {th }}$ iteration step. Although there are significant errors of some of the fit-values the iteration shrinks them within a few iterations dramatically. The overall error, defined as the value sum over the deviations between iteration result and truth of all 21 parameters is displayed in Fig. 2 for each iteration step.

\section{MEASUREMENT SETUP AND EXAMPLES}

The various delay line lengths have been realized by building two adaptor systems sliding in two fixed waveguides. Their design is not critical (beside the supression of transverse polarizations) since they have to be calibrated anyhow. They are driven by stepping motors with spindles that allow for a nominal position resolution of $6.25 \mu \mathrm{m}$ and a shift of $0.35 \mathrm{~m}$. The RF equipment consists of a HP8753C-6 GHz-network analyzer, connected with the adaptors using phase-stabilized flexible RF cables. The components are computer controlled. The adaptors and the test device are insulated to reduce thermal drifts.

A typical result of an adaptor calibration is shown in Fig. 3. It was found at $4.50 \mathrm{GHz}$ with three propagating modes $\left(\mathrm{TE}_{11}, \mathrm{TM}_{01}, \mathrm{TE}_{21}\right)$ in a $78 \mathrm{~mm}$ diameter circular waveguide. Fig. 4 demonstrates that the input reflection $\Gamma(\mathrm{L})$ (line) of the adaptor calculated with these Sparameters fits the measured dots very well. Fig. 5 gives an example for results of a device measurement.

\section{CONCLUSION AND OUTLOOK}

A method that expands network analyzer functionality in the regime of multimoded waveguides has been successfully demonstrated for up to three non-degenerated propagating modes. It provides all information needed to describe the behaviour of a given waveguide insertion at an arbitrary frequency point (or a spectrum of them). Thus it allows to predict the RF properties of every chain of known insertions, which is important not only for accelerator sections with respect to (HOM-) fields but for all waveguide applications with excitation of more than the fundamental mode. Further investigation will be spent in the resolution of (esp. polarizational) degenerated modes in order to expand the method's range of applications.

\section{SUPPORT}

Work supported by DESY and BMBF; contract 060f359.

\section{REFERENCES}

[1] H.-W. Glock, P. Hülsmann et. al.: Energy Propagation through the TESLA Channel: Measurements with Two Waveguide Modes (TESLA-Rep. 96-07), DESY, June 1996

[2] H.-W. Glock, F. Marhauser et. al.: Measurement of HOMPropagation through Cavity Chains in Terms of S-Parameters, Proc. 18th Int. Linear Accelerator Conf., Geneva, 26-30 Aug. 1996, pp. 716

\begin{tabular}{|lc|c|c|}
\hline Parm. & Truth & First Fit & 4th Iteration \\
\hline$c[1,1]$ & $0.1445+0.1334 \mathrm{I}$ & $0.3185-0.2865 \mathrm{I}$ & $0.1445+0.1334 \mathrm{I}$ \\
\hline $\mathrm{c}[2,2]$ & $-0.1162-0.1434 \mathrm{I}$ & $-0.4127-0.1202 \mathrm{I}$ & $-0.1161-0.1434 \mathrm{I}$ \\
\hline $\mathrm{c}[2,6]$ & $0.04041-0.06209 \mathrm{I}$ & $0.04047-0.06113 \mathrm{I}$ & $0.04041-0.06209 \mathrm{I}$ \\
\hline $\mathrm{c}[5,6]$ & $0.2104-0.09209 \mathrm{I}$ & $0.1841-0.1167 \mathrm{I}$ & $0.2104-0.09192 \mathrm{I}$ \\
\hline
\end{tabular}

Tab. 1: Sample S-parameters in simulated experiment

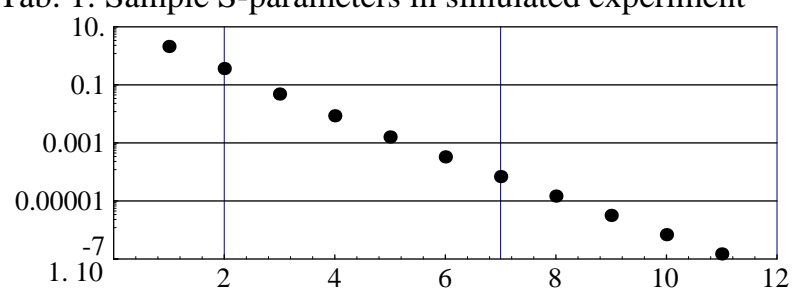

Fig. 2: Value sum of error of all S-parameters over iteration number. First point is result of fit procedure and used as start value.

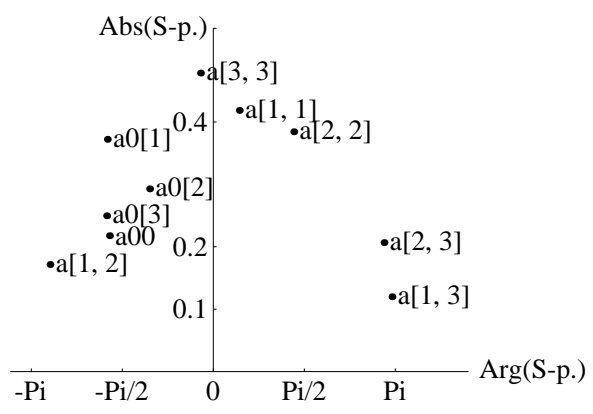

Fig. 3: Typical S-parameter of adaptor, found at $4.5 \mathrm{GHz}$
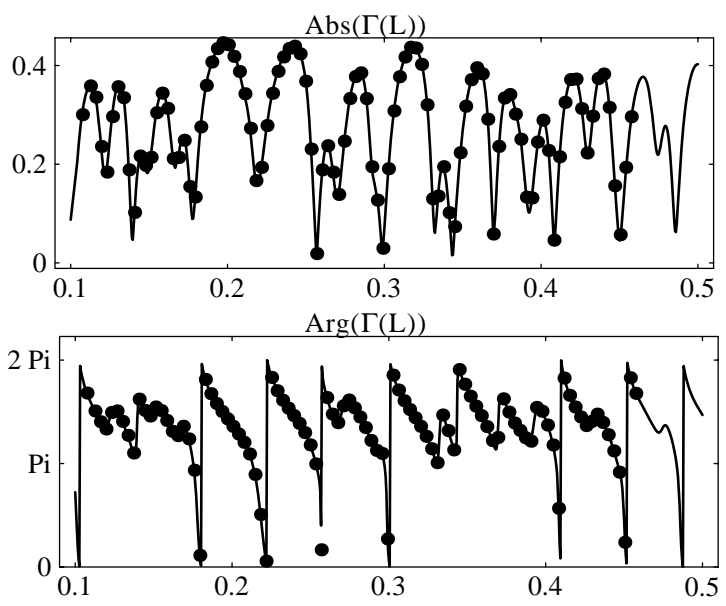

Fig. 4: $\Gamma(\mathrm{L})$, calculated from the S-parameters in Fig. 3 (line) and measured (dots)

$$
\begin{aligned}
& \text { Abs(S-p.) } \\
& \begin{array}{lr|r}
\mathrm{c}[1,3] & 1 & \\
& 0.8 & \mathrm{c}[2,4]
\end{array} \\
& 0.6 \text { c [2, 2] } \\
& \begin{array}{l|l}
0.6 & { }_{\mathrm{c}}[4,4] \\
&
\end{array}
\end{aligned}
$$

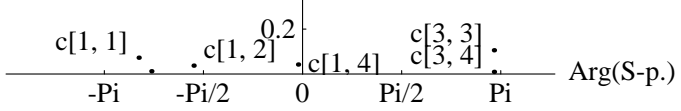

Fig. 5: Example set of S-parameters of a TESLA 9-cell copper cavity, found at $3.50 \mathrm{GHz}$ with comparably high transmission in both modes (c[1,3], c[2,4]). 